\title{
Line Source Scattering by Buried Perfectly Conducting Circular Cylinders
}

\author{
F. Frezza, ${ }^{1}$ L. Pajewski, ${ }^{2}$ C. Ponti, ${ }^{2}$ and G. Schettini ${ }^{2}$ \\ ${ }^{1}$ Department of Information Engineering, Electronics and Telecommunications, Sapienza University, \\ Via Eudossiana 18, 00184 Rome, Italy \\ ${ }^{2}$ Department of Applied Electronics, Roma Tre University, Via della Vasca Navale 84, 00146 Rome, Italy
}

Correspondence should be addressed to C. Ponti, cponti@uniroma3.it

Received 2 March 2012; Revised 17 April 2012; Accepted 18 April 2012

Academic Editor: Francesco Soldovieri

Copyright () 2012 F. Frezza et al. This is an open access article distributed under the Creative Commons Attribution License, which permits unrestricted use, distribution, and reproduction in any medium, provided the original work is properly cited.

\begin{abstract}
A two-dimensional scattering problem of a line source by a set of perfectly conducting circular cylinders buried in a semi-infinite medium is solved, in both TE and TM polarization. A cylindrical-wave approach is used and applied to both the field emitted by the source and the field scattered by the buried objects. Reflection and transmission of such fields through the planar interface are evaluated making use of the plane-wave spectrum of a cylindrical wave. Numerical results are presented, with checks confirming the validity of the method.
\end{abstract}

\section{Introduction}

Analytical and numerical techniques, to solve direct scattering by buried objects, offer useful tools in postprocessing of measured data from environmental sensing, detection of land mines, or of buried utilities, inspection of roads' and buildings' anomalies. Several methods are developed in the literature to solve such a scattering problem with cylinders buried below a planar interface [1-4] or embedded in a dielectric slab [5-9]. In the mentioned works, the excitation is given by a plane wave, which, propagating in a vacuum half-space, impinges on the interface with the medium hosting the objects. Less attention has been devoted to alternative kinds of excitations, with a more direct link to sources used in practical applications. As far as groundpenetrating radar investigation is considered, in most cases the excitation field is emitted by a dipole antenna [10] Therefore, modelling such a field with a point or a line source is a more realistic assumption. In the work of D'Yakonov [11], an analytical solution to the scattering of a time-varying electromagnetic field from a point source, by a cylindrical inhomogeneity below the surface of the earth, is developed. It is assumed that the field produced by the source is parallel to the axis of the cylinder and it is constant along it. D'Yakonov's exact solution has been extended by Ogunade
[12] to get numerical results for the case of an embedded circular cylinder excited by a line current. The problem of scattering of a subterranean cylindrical inhomogeneity from a line source is solved by Howard [13] with a modified modematching method in conjunction with an integral equation.

In [4], the cylindrical wave approach has been used to solve scattering of a plane wave by perfectly conducting cylindrical objects buried in a dielectric half-space. The method, which is based on expansions into cylindrical waves of the fields scattered by the buried cylinders, allows to take into account in an accurate way the scatterers' geometry. Moreover, making use of the plane-wave spectrum of a cylindrical wave [14], reflection and transmission through the planar interface between air and ground of the scattered fields, can also be evaluated.

In this paper, in reply to a need of realistic description of the scattering scenario, the interaction of a line source with buried perfectly conducting cylinders is dealt with. As the geometry of the problem is two-dimensional, the field radiated by the source can be described by a cylindrical wave. Therefore, the CWA presented in [4] is here suitably extended to evaluate reflection in the source half-space, the transmission in the ground, and the interaction with the buried objects. In particular, reflected and transmitted cylindrical waves of zeroth order are defined, which have been 
numerically evaluated in order to implement the theoretical analysis into a Fortran code.

The paper is organized as follows: in Section 2, the theoretical analysis is presented in detail, while in Section 3 some hints on the numerical implementation are given, and numerical results both in near- and far-field regions are reported.

\section{Theoretical Analysis}

The geometry of the problem is depicted in Figure 1: $N$ perfectly conducting circular cylinders of infinite extension along the $y$-axis are buried in a dielectric half-space of permittivity $\varepsilon_{1}=\varepsilon_{0} n_{1}^{2}$, placed below a half-space of permittivity $\varepsilon_{0}$. In the upper medium, a source given by a line of constant electric or magnetic current, of infinite extension and parallel to the planar interface and the cylinders' axes, is placed. A main reference frame $\operatorname{MRF}(O, \xi, \zeta)$ with normalized coordinates $\xi=k_{0} x$ and $\zeta=k_{0} z$, where $k_{0}=2 \pi / \lambda_{0}$ is the propagation constant in medium 0 , is introduced. The source has axis centred in $\left(\chi_{L}, \eta_{L}\right)$ in MRF; with $\left(O_{L}, \xi_{L}, \zeta_{L}\right)$ standing for the reference frame $\mathrm{RF}_{L}$ centered on the line source, the following change of coordinates applies

$$
\begin{aligned}
& \xi=\xi_{L}+\chi_{L} \\
& \zeta=\zeta_{L}+\eta_{L}
\end{aligned}
$$

where $\chi_{L}<0$.

Moreover, a reference frame $\mathrm{RF}_{q}$ centered on the axis of the $q$ th cylinder $(q=1, \ldots, N)$ is employed, both rectangular $\left(O, \xi_{q}, \zeta_{q}\right)$ and polar $\left(O, \rho_{q}, \theta_{q}\right)$, with $\xi_{q}=k_{0} x_{q}=\xi-\chi_{q}, \zeta_{q}=$ $k_{0} z_{q}=\zeta-\eta_{q}$, and $\rho_{q}=k_{0} r_{q}$, with $\left(\chi_{q}, \eta_{q}\right)$ being the coordinates in MRF of the center of the $q$ th cylinder, with radius $\alpha_{q}=k_{0} a_{q}$.

The problem is solved by means of a scalar function $V(\xi, \zeta)$, which stands for the total field component parallel to the cylinders' axes, that is, the electric field $E_{y}$ or the magnetic field $H_{y}$, in TM and TE polarization, respectively. Moreover, the field $V(\xi, \zeta)$ is decomposed in each medium into a certain number of terms, that is, the excitation field $V_{i}(\xi, \zeta)$ from the line source, and the terms following its interaction with the interface and the cylinders. In particular, the interaction of the field $V_{i}(\xi, \zeta)$ with the planar interface, in absence of the cylinders, gives a reflected field $V_{r}(\xi, \zeta)$ in medium 0 , and a transmitted field $V_{t}(\xi, \zeta)$ in medium 1 , as shown in Figure 2(a). Taking into account the geometry of the source, the incident field $V_{i}$ is here described by a cylindrical wave centered on $\left(\chi_{L}, \eta_{L}\right)$, and the reflected and transmitted fields are determined by means of the plane-wave spectrum of a cylindrical wave. From the interaction of the transmitted field with the buried cylinders, a field $V_{s}(\xi, \zeta)$ is scattered in medium 1, and the scattered-reflected and scatteredtransmitted fields $V_{\text {sr }}(\xi, \zeta)$ and $V_{\text {st }}(\xi, \zeta)$ are excited, due to reflection and transmission of $V_{s}(\xi, \zeta)$ through the interface, respectively (Figure $2(\mathrm{~b})$ ). Scattered field contributions are expressed as a sum of cylindrical waves, and the plane-wave spectrum is used to cope with reflection and transmission of such waves.

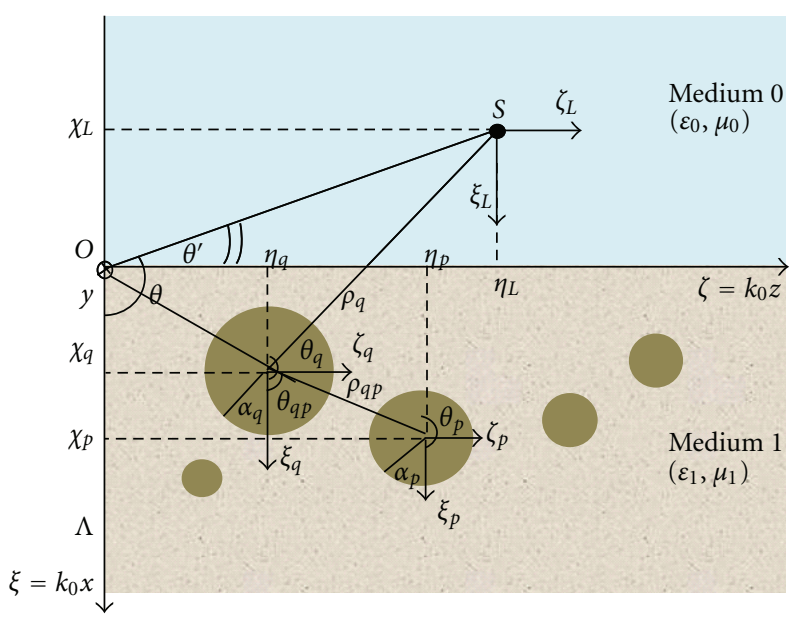

Figure 1: Geometry of the problem.

The line source radiates a $y$-directed field expressed through a Hankel function of first kind and zeroth order, with argument proportional to the distance from the source to the observation point. If the source is placed as in Figure 1, the field generated in absence of the planar interfaces and the cylinders can be written as [15]

$$
V_{i}(\xi, \zeta)=-V_{0} H_{0}^{(1)}\left(n_{0} \sqrt{\left(\xi-\chi_{L}\right)^{2}+\left(\zeta-\eta_{L}\right)^{2}}\right),
$$

where $n_{0}$ is the refraction index of the upper medium. In order to obtain the reflected and transmitted fields $V_{r}(\xi, \zeta)$ and $V_{t}(\xi, \zeta)$, respectively, the cylindrical function $\mathrm{CW}_{0}=$ $H_{0}^{(1)}$ can be expressed as a spectrum of plane waves [14]:

$$
\mathrm{CW}_{0}\left(\xi_{L}, \zeta_{L}\right)=\frac{1}{2 \pi} \int_{-\infty}^{+\infty} F_{0}\left(\xi_{L}, n_{\|}\right) e^{i n_{\|} \zeta_{L}} d n_{\|},
$$

with $n_{\|}$being the parallel component with respect to the $z$ axis of the generic plane wave of the spectrum.

In (3), the spectrum $F_{0}$ evaluated in $\xi_{L}>0$ is

$$
F_{0}\left(\xi_{L}, n_{\|}\right)=\frac{2 e^{i \xi_{L} \sqrt{1-n_{\|}^{2}}}}{\sqrt{1-n_{\|}^{2}}} .
$$

The reflected field $V_{r}(\xi, \zeta)$ is proportional to a reflected cylindrical function of zeroth order $\mathrm{RW}_{0}(\xi, \zeta)$ :

$$
V_{r}(\xi, \zeta)=-V_{0} \mathrm{RW}_{0}(\xi, \zeta),
$$

which is derived from (3), evaluating the reflected plane wave on each plane wave of the spectrum:

$$
\mathrm{RW}_{0}(\xi, \zeta)=\frac{1}{2 \pi} \int_{-\infty}^{+\infty} \Gamma_{01}\left(n_{\|}\right) F_{0}\left(-\xi-\chi_{L}, n_{\|}\right) e^{i n_{\|}\left(\zeta-\eta_{L}\right)} d n_{\|}
$$

In a similar way, the transmitted field $V_{t}(\xi, \zeta)$ is expressed through a transmitted cylindrical function of zeroth order $\mathrm{TW}_{0}(\xi, \zeta)$ :

$$
V_{t}(\xi, \zeta)=-V_{0} \operatorname{TW}_{0}(\xi, \zeta)
$$




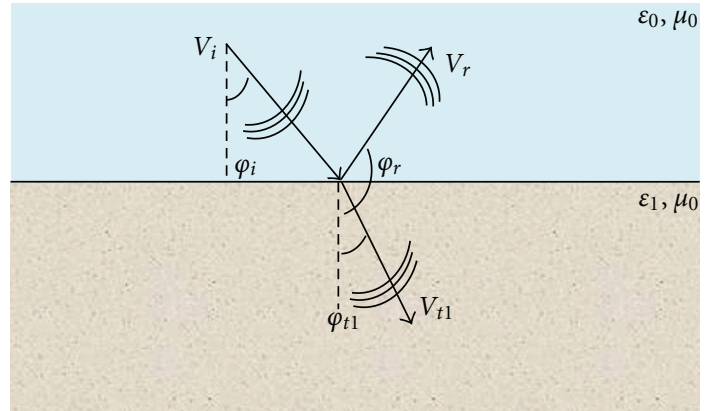

(a)

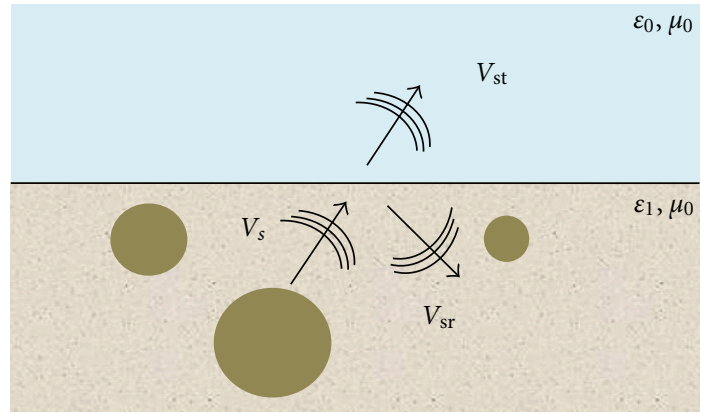

(b)

Figure 2: Decomposition of the total field: (a) incident, reflected, and transmitted cylindrical waves; (b) scattered fields.

obtained by evaluating the transmission on each plane wave of the spectrum (3):

$$
\begin{aligned}
\operatorname{TW}_{0}\left(\xi, \zeta ;-\chi_{L}\right)= & \frac{1}{2 \pi} \int_{-\infty}^{+\infty} T_{01}\left(n_{\|}\right) F_{0}\left(-\chi_{L}, n_{\|}\right) \\
& \times e^{i n_{1} \sqrt{1-\left(n_{\|} / n_{1}\right)^{2}} \xi} \mathrm{e}^{i n_{\|}\left(\zeta-\eta_{L}\right)} d n_{\|} .
\end{aligned}
$$

An expression of the transmitted field in (7) as a function of polar coordinates centred on the $p$ th cylinder's axis has to be employed, in order to impose boundary conditions on the surface of each cylinder in an easier way. For this purpose, (7) can be written in the following form:

$$
\begin{aligned}
\operatorname{TW}_{0}\left(\xi_{p}, \zeta_{p} ;-\chi_{L}\right)= & \frac{1}{2 \pi} \int_{-\infty}^{+\infty} T_{01}\left(n_{\|}\right) F_{0}\left(-\chi_{L}, n_{\|}\right) \\
& \times e^{i n_{1} \sqrt{1-\left(n_{\|} / n_{1}\right)^{2}} \chi_{P}} e^{i n_{\|}\left(\eta_{P}-\eta_{L}\right)} \\
& \times e^{i n_{1}\left(n_{\perp} \xi_{P}+n_{\|} \zeta_{P}\right)} d n_{\|},
\end{aligned}
$$

where the exponential $e^{i n_{1}\left(n_{\perp} \xi_{p}+n_{\|} \zeta_{p}\right)}$ is a transmitted planewave in $\mathrm{RF}_{P}$, which can be expanded into a series of Bessel functions:

$$
e^{i n_{1}\left(n_{\perp} \xi_{P}+n_{\|} \zeta_{P}\right)}=\sum_{\ell=-\infty}^{+\infty} i^{\ell} e^{-i \ell \varphi_{t}} J_{\ell}\left(n_{1} \rho_{P}\right) e^{i \ell \theta_{P}}
$$

In (10), $\varphi_{t}=\arctan \left[n_{\|} / \sqrt{1-\left(n_{\|} / n_{1}\right)^{2}}\right]$ is the angle of transmission of the generic plane wave of the spectrum, and the final expression of the transmitted field is

$$
\begin{aligned}
V_{t}\left(\xi_{p}, \zeta_{p}\right)= & -V_{0} \sum_{\ell=-\infty}^{+\infty} i^{\ell} J_{\ell}\left(n_{1} \rho_{P}\right) e^{i \ell \theta_{P}} \\
& \times \frac{1}{2 \pi} \int_{-\infty}^{+\infty} T_{01}\left(n_{\|}\right) F_{0}\left(-\chi_{L}, n_{\|}\right) e^{i n_{1} \sqrt{1-\left(n_{\|} / n_{1}\right)^{2}} \chi_{P}} \\
& \times e^{i n_{\|}\left(\eta_{P}-\eta_{L}\right)} e^{-i \ell \arctan \left[n_{\|} / \sqrt{1-\left(n_{\|} / n_{1}\right)^{2}}\right]} d n_{\|} .
\end{aligned}
$$

The field in (11) can also be written in the following more compact form:

$$
\begin{aligned}
V_{t}\left(\xi_{p}, \zeta_{p}\right)= & -V_{0} \sum_{\ell=-\infty}^{+\infty} i^{\ell} J_{\ell}\left(n_{1} \rho_{P}\right) e^{i \ell \theta_{P}} \\
& \times \operatorname{TW}_{0, \ell}\left(\chi_{P}, \eta_{P}-\eta_{L},-\chi_{L}\right) .
\end{aligned}
$$

As to the scattered fields $V_{s}(\xi, \zeta), V_{\text {sr }}(\xi, \zeta)$, and $V_{\text {st }}(\xi, \zeta)$, the same expressions derived in [4] are used. The scattered field $V_{s}(\xi, \zeta)$ is an expansion into cylindrical functions with unknown coefficients $c_{q m}$ :

$$
V_{s}(\xi, \zeta)=V_{0} \sum_{q=1}^{N} \sum_{m=-\infty}^{+\infty} c_{q m} \mathrm{CW}_{m}\left(n_{1} \xi_{q}, n_{1} \zeta_{q}\right),
$$

with $\mathrm{CW}_{m}\left(n_{1} \xi_{q}, n_{1} \zeta_{q}\right)=H_{m}^{(1)}\left(n_{1} \rho_{q}\right) e^{i \ell \theta_{q}}$, where $H_{m}^{(1)}\left(n_{1} \rho_{q}\right)$ is the first-kind Hankel function of order m. Making use of the addition theorem of Hankel functions, the field in (13) can be written in $\mathrm{RF}_{q}$, as follows:

$$
\begin{aligned}
& V_{s}\left(\xi_{q}, \zeta_{q}\right)= V_{0} \sum_{\ell=-\infty}^{\infty} J_{\ell}\left(n_{1} \rho_{p}\right) e^{i \ell \theta_{p}} \sum_{q=1}^{N} \sum_{m=-\infty}^{+\infty} i^{m} c_{q m} \\
& \times {\left[\operatorname{CW}_{m-\ell}\left(n_{1} \xi_{q p}, n_{1} \zeta_{q p}\right)\left(1-\delta_{q p}\right)\right.} \\
&\left.+\frac{H_{\ell}^{(1)}\left(n_{1} \rho_{p}\right)}{J_{\ell}\left(n_{1} \rho_{p}\right)} \delta_{q p} \delta_{\ell m}\right]
\end{aligned}
$$

To express the scattered fields $V_{\mathrm{sr}}(\xi, \zeta)$ and $V_{\mathrm{st}}(\xi, \zeta)$, the cylindrical function $\mathrm{CW}_{m}$ is expanded into a Fourier spectrum $F_{m}$ :

$$
\mathrm{CW}_{m}(\xi, \zeta)=\frac{1}{2 \pi} \int_{-\infty}^{+\infty} F_{m}\left(\xi, n_{\|}\right) e^{i n_{\|} \zeta} d n_{\|},
$$

and the explicit expression of the spectrum is given by

$$
F_{m}\left(\xi, n_{\|}\right)=\frac{2 e^{i|\xi| \sqrt{1-n_{\|}^{2}}}}{\sqrt{1-n_{\|}^{2}}} \begin{cases}e^{-i m \arccos n_{\|}}, & \xi \geq 0, \\ e^{i m \arccos n_{\|}}, & \xi \leq 0 .\end{cases}
$$


The scattered-reflected field $V_{\mathrm{sr}}(\xi, \zeta)$ is the sum of the fields scattered by each cylinder and reflected by the interface. In particular, reflected cylindrical waves are defined as basis functions of the scattered-reflected field, which are obtained by evaluating the reflection on each plane wave of the spectrum (16):

$$
\begin{aligned}
\mathrm{RW}_{m} & \left(n_{1} \xi, n_{1} \zeta\right) \\
& =\frac{1}{2 \pi} \int_{-\infty}^{+\infty} \Gamma_{10}\left(n_{\|}\right) F_{m}\left(n_{1} \xi, n_{\|}\right) e^{i n_{1} n_{\|} \zeta} d n_{\|} .
\end{aligned}
$$

Thus, the scattered-reflected field is

$$
V_{\mathrm{sr}}(\xi, \zeta)=V_{0} \sum_{q=1}^{N} \sum_{m=-\infty}^{+\infty} c_{q m} \mathrm{RW}_{m}\left[n_{1}\left(-2 \chi_{q}-\xi_{q}\right), n_{1} \zeta_{q}\right] .
$$

An expression of the scattered field in medium 1, which is a function of polar coordinates centred on the $p$ th cylinder, is used when boundary conditions are imposed:

$$
\begin{aligned}
V_{\mathrm{sr}}(\xi, \zeta)= & V_{0} \sum_{\ell=-\infty}^{+\infty} J_{\ell}\left(n_{1} \rho_{p}\right) e^{i \ell \theta_{p}} \sum_{q=1}^{N} \sum_{m=-\infty}^{+\infty} c_{q m} \mathrm{RW}_{m+\ell} \\
& \times\left[-n_{1}\left(\chi_{q}+\chi_{p}\right), n_{1}\left(\eta_{q}-\eta_{p}\right)\right] .
\end{aligned}
$$

Equation (19) is obtained from (18), making use of (16) and (17), and of the expansion of a plane wave into Bessel functions.

Transmitted cylindrical waves are defined as basis functions of the scattered-transmitted field:

$$
\begin{aligned}
\operatorname{TW}_{m}(\xi, \zeta, \chi)= & \frac{1}{2 \pi} \int_{-\infty}^{+\infty} T_{10}\left(n_{\|}\right) F_{m}\left(-n_{1} \chi, n_{\|}\right) \\
& \times e^{-i \sqrt{1-\left(n_{1} n_{\|}\right)^{2}}(\xi+\chi)} e^{i n_{1} n_{\|} \zeta} d n_{\|},
\end{aligned}
$$

which is defined as follows:

$$
V_{\text {st }}(\xi, \zeta)=V_{0} \sum_{q=1}^{N} \sum_{m=-\infty}^{+\infty} c_{q m} \operatorname{TW}_{m}\left(\xi_{q}, \zeta_{q}, \chi_{q}\right) .
$$

Under the hypothesis of perfectly conducting cylinders, boundary conditions of zero electric field on the cylinders' surface are imposed, which are

$$
V_{t}+V_{s}+\left.V_{s r}\right|_{\rho_{p}=\alpha_{p}}=0
$$

in TM polarization, and

$$
\left.\frac{\partial}{\partial \rho_{p}}\left(V_{t}+V_{s}+V_{\mathrm{sr}}\right)\right|_{\rho_{p}=\alpha_{p}}=0
$$

in TE polarization.

From boundary conditions (22) and (23), a linear system in the unknown expansion coefficients $c_{q m}$ is derived:

$$
\begin{aligned}
& \sum_{q=1}^{N} \sum_{m=-\infty}^{+\infty} A_{q p}^{\ell m(\mathrm{TM}, \mathrm{TE})} c_{q m}=B_{p}^{\ell(\mathrm{TM}, \mathrm{TE})} \\
& p=1, \ldots, N, \quad \ell=0, \pm 1, \ldots, \pm \infty,
\end{aligned}
$$

with

$$
\begin{aligned}
& A_{q p}^{\ell m(\mathrm{TM}, \mathrm{TE})}= i^{-\ell} G_{\ell}^{(\mathrm{TM}, \mathrm{TE})}\left(n_{1} \rho_{p}\right) \\
& \times\left\{\mathrm{CW}_{m-\ell}\left(n_{1} \xi_{q p}, n_{1} \zeta_{q p}\right)\left(1-\delta_{q p}\right)\right. \\
&+\mathrm{RW}_{m+\ell}\left[-n_{1}\left(\chi_{q}+\chi_{p}\right), n_{1}\left(\eta_{q}-\eta_{p}\right)\right] \\
&\left.+\frac{\delta_{q p} \delta_{\ell m}}{G_{\ell}^{(\mathrm{TM}, \mathrm{TE})}\left(n_{1} \rho_{p}\right)}\right\}, \\
& B_{p}^{\ell(\mathrm{TM}, \mathrm{TE})}=-G_{\ell}^{(\mathrm{TM}, \mathrm{TE})}\left(n_{1} \rho_{p}\right) \mathrm{TW}_{0, \ell}\left(\chi_{p}, \eta_{p}-\eta_{L},-\chi_{L}\right),
\end{aligned}
$$

where $G_{\ell}^{(\mathrm{TM})}(\cdot)=J_{\ell}(\cdot) / H_{\ell}^{(1)}(\cdot)$ and $G_{\ell}^{(\mathrm{TE})}(\cdot)=J_{\ell}^{\prime}(\cdot) / H_{\ell}^{\prime(1)}(\cdot)$.

\section{Numerical Results}

The numerical implementation of the analytical method presented in Section 2 has been performed according to criteria of accuracy. As to solve system (24), a truncation on the cylindrical orders given by $m$ and $\ell$ to a finite number of terms is needed, and the rule $M_{t}=\left\lfloor 3 n_{1} \alpha\right\rfloor$ [16], with $\alpha$ being the radius of the largest cylinder, has been employed, with a good compromise between accuracy and computational heaviness. A numerical evaluation of Cylindrical Functions relevant to the spectral integrals (6), (8), (17), and (20) is also needed. Suitable algorithms have been developed in [17] for the evaluation of Reflected Cylindrical Functions $\mathrm{RW}_{m}$ defined in (18), and they have been generalized in [18] to the evaluation of Transmitted Cylindrical Functions $\mathrm{TW}_{m}$ reported in (20). The developed algorithms take into account the infinite extension of the integration domain, and they detect where the spectrum of each wave function is oscillating and evanescent, applying a different integration procedure. On oscillating integrands, a further decomposition of the integration domain is applied making use of adaptive techniques, and Gaussian quadrature rules are employed. As to the evanescent part of the spectrum, it is decomposed into a suitable number of subintervals, where fixed low-order Gauss-Legendre rules are applied.

The Reflected Cylindrical Function of zeroth order $\mathrm{RW}_{0}$ in (6) can be easily evaluated applying the algorithm developed in [17], while a modified integration algorithm has been developed for the evaluation of the Transmitted Cylindrical Functions of zeroth order $\mathrm{TW}_{0}$ in (8). With the transmitted field expressed in a cylindrical frame centred on the axis of the $p$ th cylinder, as in (11), the $\mathrm{TW}_{0}$ is expressed as follows:

$$
\begin{aligned}
\operatorname{TW}_{0}\left(\chi_{p}, \eta_{p}-\eta_{L} ;-\chi_{L}\right) \\
=\frac{1}{2 \pi} \int_{-\infty}^{+\infty} T_{01}\left(n_{\|}\right) F_{0}\left(-\chi_{L}, n_{\|}\right)
\end{aligned}
$$




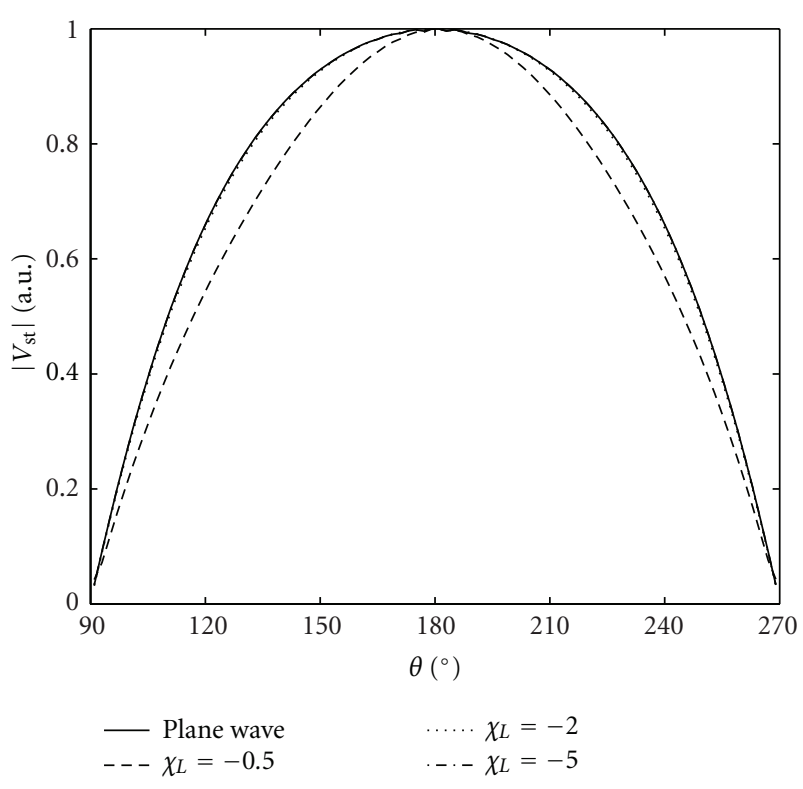

FIGURE 3: Scattered far-field diagram of a perfectly conducting cylinder $\left(\alpha=1, \chi=2.57, \eta=0, n_{1}=2\right.$, TM polarization) by a line source centred on $\eta_{L}=0$, and placed at different positions $\chi_{L}$; results are compared to the scattered field by a plane-wave excitation.

$$
\begin{aligned}
& \times e^{i n_{1} \sqrt{1-\left(n_{\|} / n_{1}\right)^{2}} \chi_{P}} e^{i n_{\|}\left(\eta_{P}-\eta_{L}\right)} \\
& \times e^{-i \ell \arctan \left[n_{\|} / \sqrt{1-\left(n_{\|} / n_{1}\right)^{2}}\right]} d n_{\|} .
\end{aligned}
$$

Comparing (26) to (20), the additional term $e^{-i \ell \arctan \left[n_{\|} / \sqrt{1-\left(n_{\|} / n_{1}\right)^{2}}\right]}$ appears in (26). Therefore, a different decomposition and evaluation have been applied, following the general rules of the algorithm in [18].

Some checks of convergence have been performed on the implemented numerical code, as the one reported in Figure 3, where the scattered far field from a plane-wave excitation is compared to the one from a line source, which is placed at an increasing distance from the interface. A perfectly conducting cylinder of normalized radius $\alpha=1$, centered in $\chi=2.57$ and $\eta=0$, has been simulated. The refractive index of the lower medium is $n_{1}=2$, and the polarization state is TM. The line source has been centred on $\eta_{L}=0$, and placed at three different distances $\chi_{L}=$ $-0.5,-2,-5$ from the interface. A good convergence to the scattered field by a plane-wave excitation can be observed, as the cylindrical wave front is moved away from the interface. In particular, in the cases $\chi_{L}=-2$ and $\chi_{L}=-5$ the curves are superimposed to the plane wave results. Accuracy of results is shown in Figure 4, for the case of scattered field due to a line source placed in $\chi_{L}=-2$. Truncation on cylindrical functions is applied according to the rule $M_{t}=\left\lfloor 3 n_{1} \alpha\right\rfloor$, and with the given parameters it yields $M_{t}=6$. Scatteredtransmitted field is then evaluated increasing the order $M_{t}$ to $M_{t}+1=7$ and $M_{t}+2=8$. In Figure 4 , the absolute value of the difference between scattered-transmitted fields evaluated

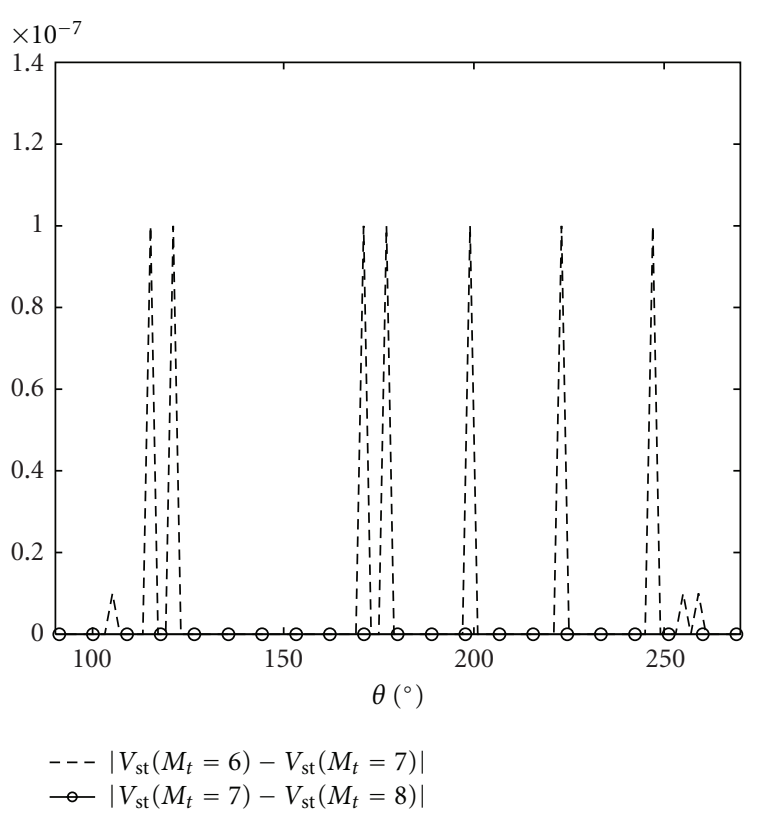

FIGURE 4: Check of accuracy on results in Figure 3, in the case of line source in $\chi_{L}=-2$, increasing the truncation order on cylindrical functions $M_{t}$.

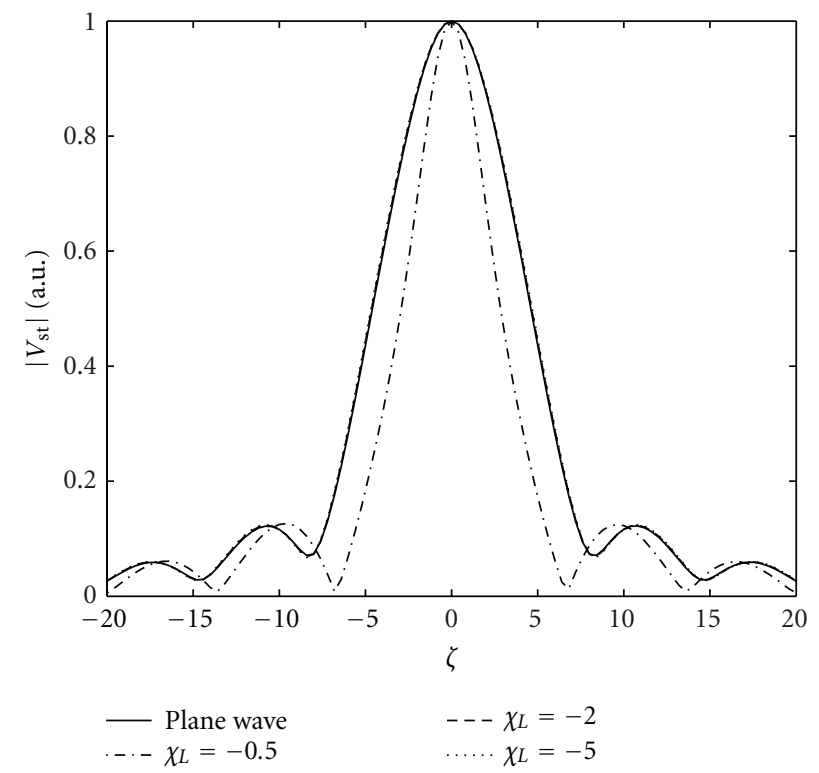

FIGURE 5: Scattered near-field diagram of a perfectly conducting cylinder $\left(\alpha=1, \chi=2.57, \eta=0, n_{1}=2\right.$, TM polarization) by a line source centred on $\eta_{L}=0$ and placed at different positions $\chi_{L}$. The field is evaluated at $\xi=-1$, and results are compared to the scattered field by a plane-wave excitation.

for two consecutive value of $M_{t}$ is displayed, confirming the accuracy of the rule $M_{t}=\left\lfloor 3 n_{1} \alpha\right\rfloor$.

The check of convergence to plane-wave excitation is proposed also in the near field, in Figure 5. The simulated parameters are the same as in Figure 3, and the scattered field is now observed along a line parallel to the interface, placed 


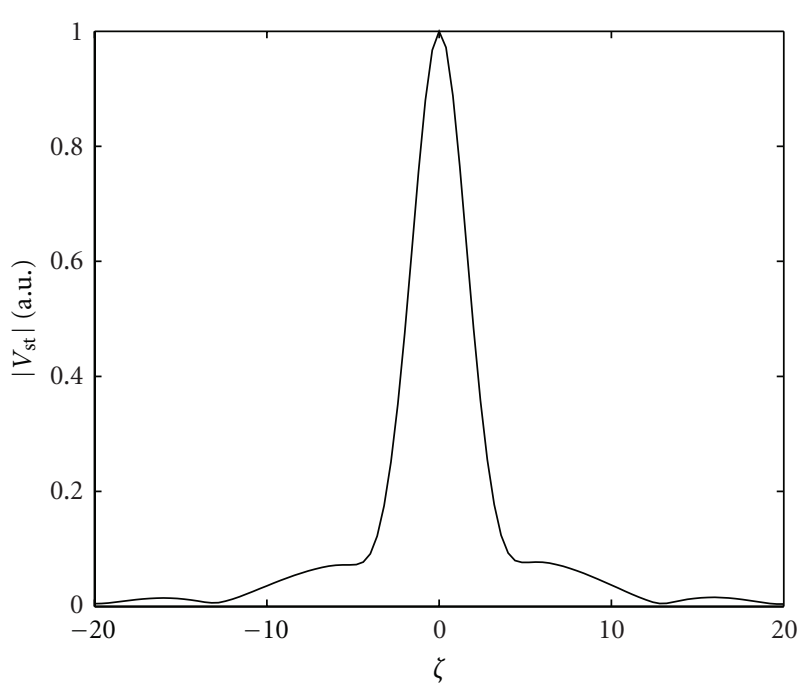

Figure 6: Scattered near-field diagram of two perfectly conducting cylinders $\left(\alpha_{1}=\alpha_{2}=1, \chi_{1}=\chi_{2}=2.57, \eta_{1}=1.5, \eta_{2}=-1.5, n_{1}=2\right.$, TM polarization) by a line source centred on $\eta_{L}=0$ and $\chi_{L}=-0.5$. Results are evaluated at the distance $\xi=-1$.

at the normalized distance $\xi=-1$. A good convergence of the curves with $\chi_{L}=-2$ and $\chi_{L}=-5$ to the plane wave one can be observed.

A case of interaction between two identical cylinders of radius $\alpha_{1}=\alpha_{2}=1$, buried at a symmetric distance of $\eta_{1,2}= \pm 1.5$ from the $\xi$-axis, at the depth $\chi_{1}=\chi_{2}=2.57$, in a medium with refraction index $n_{1}=2$, is also reported. The scattered near-field diagram, evaluated at the distance $\xi=-1$, is given in Figure 6, in the TM polarization state, with a source centred in $\eta_{L}=0$ and $\chi_{L}=-0.5$.

\section{Conclusions}

A theoretical method to solve the two-dimensional scattering of a line source by a set of perfectly conducting cylinders buried in a dielectric half-space has been presented. The CWA has been used to express both the source radiation and its interaction with the interface as well as the scattered fields by the cylinders. The method can deal with TM or TE polarization states and gives solutions both in the near and far field. Results of its numerical implementation have been presented, which have been obtained through an accurate evaluation of spectral integrals.

As future work, the presented analysis, carried out in the spectral domain, will be extended to a time-domain solution following an approach analogous to the one developed in [19] for pulsed plane-wave excitation. A possible extension of the method may also consider a lossy dielectric halfspace, extending the approach developed in [20, 21] for monochromatic plane-wave excitation. The scattered field would be represented as an expansion of Hankel's functions of complex argument. As to Reflected and Transmitted Cylindrical Functions, used both to express reflection and transmission of the incident wave and as basis functions of the scattered-reflected and scattered-transmitted fields, the general definitions presented in the lossless case are still applied, apart from replacing the real refraction index of the lower medium with a complex one. The algorithm developed in [17] for the evaluation of the spectral integrals in the lossless case may still be applied, although the complex reflection index would lead to a different behaviour of the integrand both on the evanescent and homogeneous parts of the spectrum.

\section{References}

[1] K. Hongo and A. Hamamura, "Asymptotic solutions for the scattered field of plane wave by a cylindrical obstacle buried in a dielectric half-space," IEEE Transactions on Antennas and Propagation, vol. 34, no. 11, pp. 1306-1312, 1986.

[2] C. M. Butler, X. B. Xu, and A. W. Glisson, "Current induced on a conducting cylinder located near the planar interface between two semi-infinite half spaces," IEEE Transactions on Antennas and Propagation, vol. 33, no. 6, pp. 616-624, 1985.

[3] Q. A. Naqvi, A. A. Rizvi, and Z. Yaqoob, "Corrections to asymptotic solutions for the scattered fields of plane wave by a cylindrical obstacle buried in a dielectric half-space," IEEE Transactions on Antennas and Propagation, vol. 48, no. 5, pp. 846-849, 2000.

[4] M. Di Vico, F. Frezza, L. Pajewski, and G. Schettini, "Scattering by a finite set of perfectly conducting cylinders buried in a dielectric half-space: a spectral-domain solution," IEEE Transactions on Antennas and Propagation, vol. 53, no. 2, pp. 719727, 2005.

[5] K. A. Michalski and D. Zheng, "Electromagnetic scattering and radiation by surfaces of arbitrary shape in layered media-I: theory," IEEE Transactions on Antennas and Propagation, vol. 38, no. 3, pp. 335-344, 1990.

[6] K. A. Michalski and D. Zheng, "Electromagnetic scattering and radiation by surfaces of arbitrary shape in layered media-II: implementation and results for contiguous halfspaces," IEEE Transactions on Antennas and Propagation, vol. 38, no. 3, pp. 345-352, 1990.

[7] S. C. Lee, "Light scattering by closely spaced parallel cylinders embedded in a finite dielectric slab," Journal of the Optical Society of America A, vol. 16, no. 6, pp. 1350-1361, 1999.

[8] R. Paknys, "Reflection and transmission by reinforced concrete-numerical and asymptotic analysis," IEEE Transactions on Antennas and Propagation, vol. 51, no. 10, pp. 2852-2861, 2003.

[9] F. Frezza, L. Pajewski, C. Ponti, and G. Schettini, "Scattering by perfectly conducting circular cylinders buried in a dielectric slab through the cylindrical wave approach," IEEE Transactions on Antennas and Propagation, vol. 57, no. 4, pp. 1208-1217, 2009.

[10] D. J. Daniels, Surface Penetrating Radar, IEE, London, UK, 2nd edition, 2004.

[11] B. P. D'Yakonov, “The diffraction of electromagnetic waves by a circular cylinder in a homogeneous half space," Bulletin de l'Académie des Sciences de l'URSS, vol. 9, pp. 950-955, 1959.

[12] S. O. Ogunade, "Electromagnetic response of an embedded cylinder for line current excitation," Geophysics, vol. 46, no. 1, pp. 45-52, 1981.

[13] A. Q. Howard, "The electromagnetic fields of a subterranean cylindrical inhomogeneity excited by a line source," Geophysics, vol. 37, pp. 975-984, 1972. 
[14] G. Cincotti, F. Gori, M. Santarsiero, F. Frezza, F. Furnò, and G. Schettini, "Plane wave expansion of cylidrical functions," Optics Communications, vol. 95, no. 4-6, pp. 192-198, 1993.

[15] C. A. Balanis, Advanced Engineering Electromagnetics, John Wiley \& Sons, New York, NY, USA, 1989.

[16] A. Z. Elsherbeni, "A comparative study of two-dimensional multiple scattering techniques," Radio Science, vol. 29, no. 4, pp. 1023-1033, 1994.

[17] R. Borghi, F. Frezza, M. Santarsiero, C. Santini, and G. Schettini, "A quadrature algorithm for the evaluation of a 2D radiation integral with a highly oscillating kernel," Journal of Electromagnetic Waves and Applications, vol. 14, no. 10, pp. 1353-1370, 2000.

[18] M. Di Vico, F. Frezza, L. Pajewski, and G. Schettini, "Scattering by buried dielectric cylindrical structures," Radio Science, vol. 40, no. 6, Article ID RS6S18, 2005.

[19] F. Frezza, P. Martinelli, L. Pajewski, and G. Schettini, "Shortpulse electromagnetic scattering from buried perfectly-conducting cylinders," IEEE Geoscience and Remote Sensing Letters, vol. 4, no. 4, pp. 611-615, 2007.

[20] F. Frezza, G. Schettini, and N. Tedeschi, "Generalized planewave expansion of cylindrical functions in lossy media convergent in the whole complex plane," Optics Communications, vol. 284, no. 16-17, pp. 3867-3871, 2011.

[21] F. Frezza, L. Pajewski, C. Ponti, G. Schettini, and N. Tedeschi, "Electromagnetic scattering by a metallic cylinder buried in a lossy medium with the cylindrical wave approach," IEEE Geoscience and Remote Sensing Letters, vol. 9, pp. 179-183, 2012. 

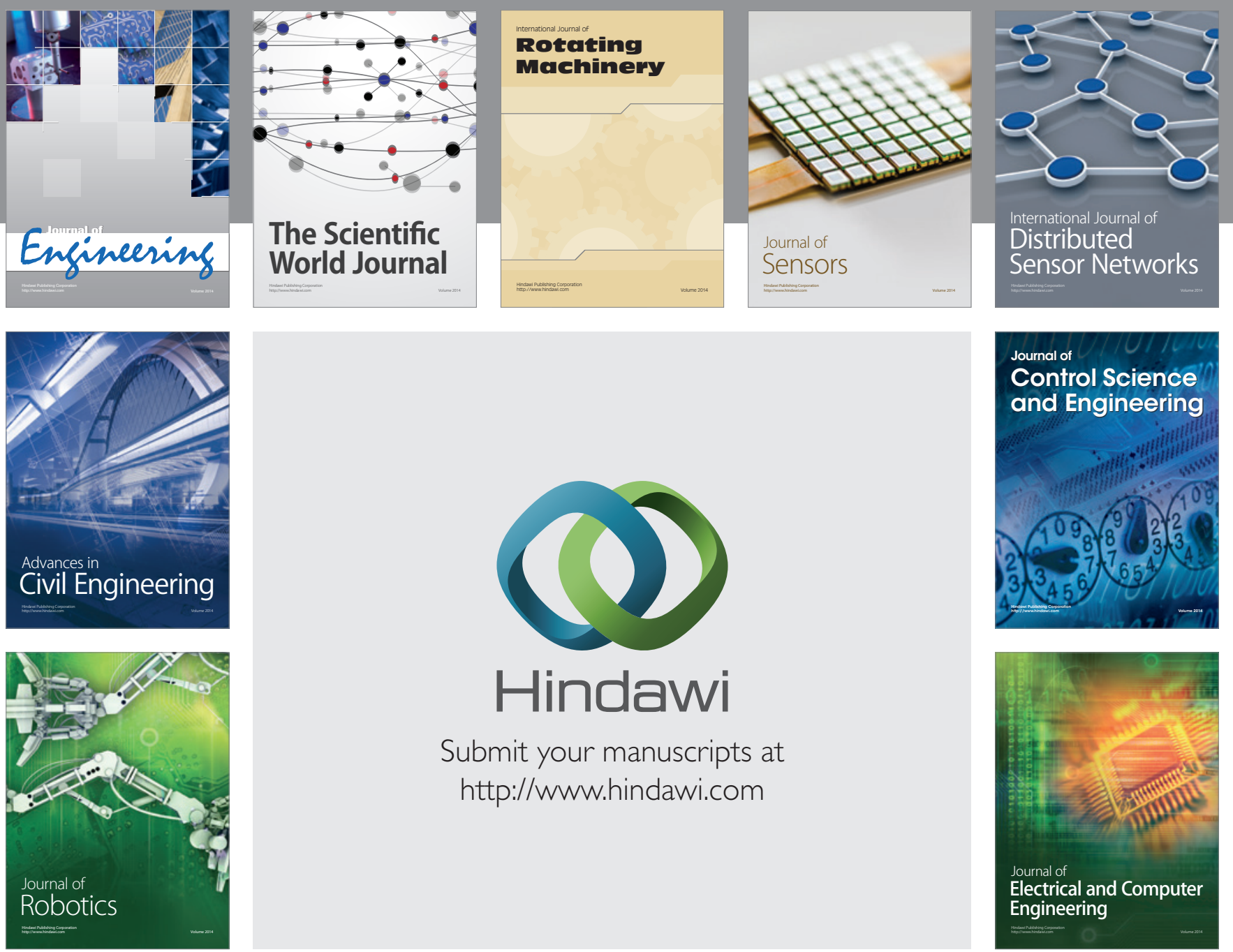

Submit your manuscripts at

http://www.hindawi.com
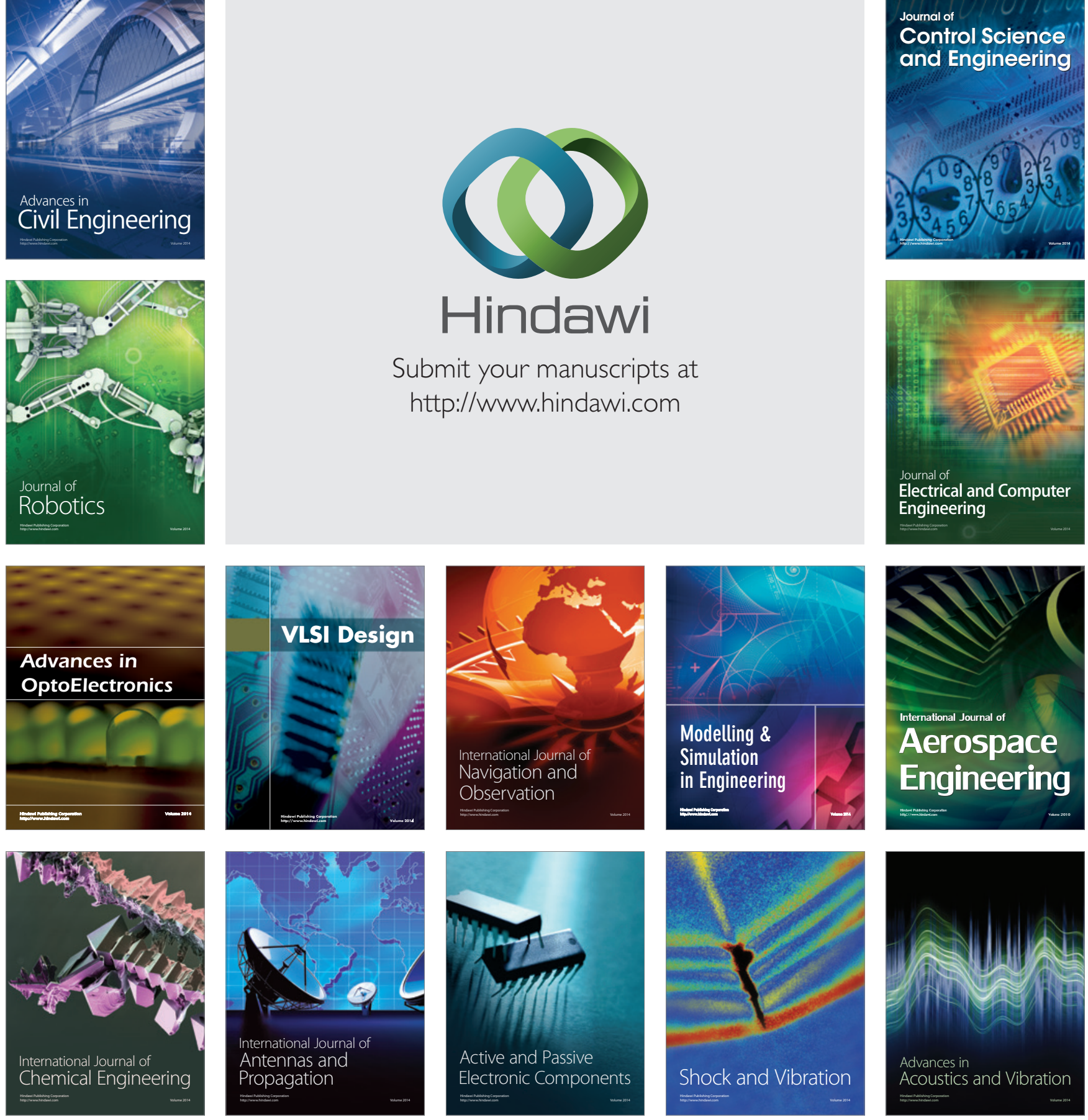